\title{
NEUTRALLY STABLE WAVE MOTIONS IN THERMALLY STRATIFIED POISEUILLE-COUETTE FLOW
}

\author{
JAMES P. DENIER ${ }^{1}$ and ANDREW P. BASSOM ${ }^{2}$
}

(Received 18 June 1996; revised 3 July 1997)

\begin{abstract}
The influence of thermal buoyancy on neutral wave modes in Poiseuille-Couette flow is considered. We examine the modifications to the asymptotic structure first described by Mureithi, Denier \& Stott [16], who demonstrated that neutral wave modes in a strongly thermally stratified boundary layer are localized at the position where the streamwise velocity attains its maximum value. The present work demonstrates that such a flow structure also holds for Poiseuille-Couette flow but that a new flow structure emerges as the position of maximum velocity approaches the wall (and which occurs as the level of shear, present as a consequence of the Couette component of the flow, is increased). The limiting behaviour of these wave modes is discussed thereby allowing us to identify the parameter regime appropriate to the eventual restabilization of the flow at moderate levels of shear.
\end{abstract}

\section{Introduction}

Our concern is with the effect of thermal buoyancy on unstably stratified PoiseuilleCouette flow between parallel plates; the ultimate aim is to describe the restabilization of the flow to two-dimensional travelling wave modes as the degree of basic shear (due to the Couette component of the flow) is increased.

The influence of thermal stratification on the stability of a wide variety of fluid flows is of considerable practical interest with areas of applicability ranging from the design of efficient heat exchangers to the problem of free convection in the upper mantle of the earth. There is a large body of literature on the problem of the stability of thermally stratified parallel flows, both uniform and with a component of basic shear. Gage [9] considered the effect of stable thermal stratification on the stability properties of a parallel flow and demonstrated that the flow is rendered stable by the

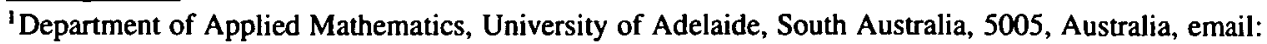
jdenier@maths.adelaide.edu.au

${ }^{2}$ Department of Mathematics, University of Exeter, North Park Road, Exeter EX4 4QE, United Kingdom, email: drew@maths.exeter.ac.uk

(C) Australian Mathematical Society, 1998, Serial-fee code 0334-2700/98
} 
application of a sufficiently large thermal gradient. This work utilized the asymptotic solutions obtained by Koppel [14] to the equations governing infinitesimally small perturbations to the basic flow and was restricted to fluids of unit Prandtl number. The problem of unstable thermal stratification was considered by Gage \& Reid [10] who, by noting the similarities between the flow of a stratified fluid with unit Prandtl number and that of the spiral flow between rotating cylinders considered earlier by Hughes \& Reid [13], were able to construct the three-dimensional stability boundary from the corresponding two-dimensional results by making use of Squire's transformation. This allowed them to delineate the regions in Rayleigh number-Reynolds number space for which the flow is susceptible to longitudinal rolls (aligned with the direction of the mean flow) or three-dimensional wave motions (which in the large Reynolds number limit evolve into Tollmien-Schlichting waves).

Further work on the stability of stratified plane Poiseuille flow can be found in Tveitereid [19] who considered flows of arbitrary Prandtl number (thus extending the results of [10]); Schäfer \& Herwig [18], who considered the effect of temperature dependent viscosity on the flow stability, and Vasilyev \& Paolucci [20], who removed the Boussinesq assumption employed in earlier studies. By considering fluids whose thermal conductivity and viscosity are governed by Sutherland's law they demonstrated that the dominant form of motion is still the longitudinal roll (aligned with the direction of the mean flow) and that the critical Rayleigh number increases as a function of the difference in the applied temperatures of the bounding plates.

The modifications to the stability properties of the flow in the presence of basic shear (as exemplified by thermally stratified Couette flow) has been considered by several authors, see for example [4] or [11]. The results of early studies in this area are summarized by Fujimura \& Kelly [8] who resolved some misconceptions that had arisen concerning the stability of stratified Poiseuille flow. By solving the eigenvalue problem spectrally using Chebyshev polynomials, Fujimura \& Kelly [8] were able to demonstrate that the effect of the imposition of a component of shear to the underlying basic flow is to increase the critical Rayleigh number of the flow. Their study was, however, restricted to small to moderate values of the Reynolds number, typically in the range $[0.01,100]$, and so was unable to consider the ultimate restabilization of the flow as the level of shear is increased. (As will be demonstrated in the present paper it is the large Reynolds number asymptotic regime that holds the key to understanding the restabilization of the flow due to increased shear.)

The large Reynolds number limit is a natural one in the context of stratified boundary layer flows and it is recent results in this area that have motivated the current investigation. Findings of Mureithi, Denier \& Stott [16], who were concerned with the effect of thermal stratification on accelerating boundary layers, demonstrated that in the limit of strong thermal coupling (between the momentum and energy fields) neutral wave modes become localized in a thin viscous layer situated at the position 
at which the streamwise velocity in the boundary layer attains its maximum. The fact that the streamwise velocity attains a maximum within the boundary layer is a direct consequence of the strong thermal coupling thus resulting in an enhanced buoyancy driven acceleration in the streamwise direction; furthermore these neutral wave modes travel at the maximum speed of the flow. In the case of thermally stratified Poiseuille flow the streamwise velocity attains a maximum at the centreline of the channel and so we anticipate that, in the large Reynolds number limit, the viscous neutral wave structure described by Mureithi et al. [16] will become relevant. However, with the introduction of basic shear (represented by a Couette component of the flow) the position of maximum streamwise velocity moves from the centreline of the channel towards the upper rigid surface. There must then be a level of shear at which the viscous structure of [16] must be adapted in order to account for the presence of the rigid boundary. The major aims of this work are to describe these modifications to the analysis of [16] and to account for the eventual re-stabilization of the flow as a function of the level of wall shear.

The outline of the paper is as follows. In Section 2 we formulate the problem and present results of a numerical solution of the equations governing small amplitude perturbations to the basic flow. The effect of increasing the level of basic shear is investigated and, in turn, this motivates the high Reynolds number asymptotic limits considered in Sections 3 and 4. Inviscid wave modes are considered in Section 3 (corresponding to the large Reynolds number- $O(1)$ wavenumber limit) and the results demonstrate that it is the large wavelength motions which are first stabilized by the basic shear. As a consequence, within Section 4 we consider the combined asymptotic limit of large Reynolds number and large wavenumber. At this level modifications to the structure described by Mureithi et al. [16] are required in order to account for the effect of basic shear. The ultimate fate of the flow as the level of shear is increased is found to be governed by a sixth order system of coupled ordinary differential equations; this system is solved numerically and enables us to predict the value of wall shear beyond which the flow can no longer support unstable travelling wave modes. Finally, in Section 5 we present some conclusions and discuss the extension of this problem to finite amplitude perturbations.

\section{Formulation}

Consider the flow of a viscous incompressible fluid of density $\rho$ and kinematic viscosity $v$ between two parallel plates situated at $y^{*}= \pm h / 2$. Suppose that the upper plate is moving with velocity $U_{0}$ and has temperature $T_{1}$ whilst the lower is moving with velocity $-U_{0}$ and has temperature $T_{0}$ (see Figure 1); in addition there is an applied streamwise pressure gradient $-D$. We nondimensionalise all distances with 
respect to the half-width $h / 2$, velocities with respect to $U_{m}=2 D /\left(v h^{2}\right)$ (the centreline speed) and temperature with respect to half the temperature difference, $\left(T_{1}-T_{0}\right) / 2$, between the upper and lower boundaries. Under the Boussinesq approximation the basic velocity and temperature fields can then be written in the form

$$
\bar{u}=1-y^{2}+u_{w} y, \quad \bar{v}=0, \quad \bar{T}=\bar{T}_{0}+y,
$$

where we have defined the dimensionless shear $u_{w}=U_{0} / U_{m}$ and $\bar{T}_{0}=\left(T_{1}+T_{0}\right) /\left(T_{1}-\right.$ $\left.T_{0}\right)$. In terms of the non-dimensional variables the upper plate moves at a velocity $u_{w}$ whilst the lower plate moves with velocity $-u_{w}$.

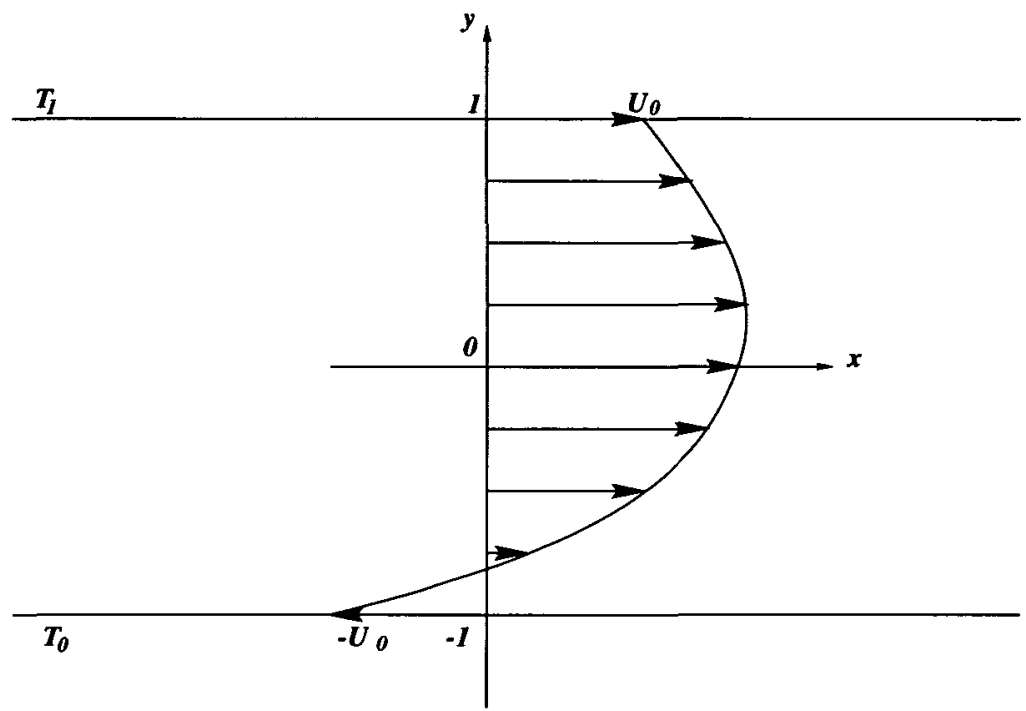

FIGURE 1. Diagram illustrating the coordinate system

The equations governing small amplitude, two dimensional, wave-like perturbations to this basic flow are then given by (see [9], [10] and [14])

$$
\begin{aligned}
\left\{\frac{\partial^{2}}{\partial y^{2}}-\alpha^{2}-i \alpha \operatorname{Re}(\bar{u}-c)\right\} u & =i \alpha \operatorname{Re} p+\operatorname{Re} \bar{u}_{y} v, \\
\left\{\frac{\partial^{2}}{\partial y^{2}}-\alpha^{2}-i \alpha \operatorname{Re}(\bar{u}-c)\right\} v & =\operatorname{Re} \frac{\partial p}{\partial y}-4 \operatorname{RiRe\theta }, \\
\left\{\frac{\partial^{2}}{\partial y^{2}}-\alpha^{2}-i \alpha \operatorname{RePr}(\bar{u}-c)\right\} \theta & =\operatorname{RePr} \bar{T}_{y} v, \\
i \alpha u+\frac{\partial v}{\partial y} & =0
\end{aligned}
$$


together with the boundary conditions

$$
u=v=T=0 \quad \text { on } \quad y= \pm 1 .
$$

Here $u, v, \theta$ and $p$ are the streamwise velocity, normal velocity, temperature and pressure perturbations respectively; we have assumed that the perturbations are proportional to $\exp [i \alpha(x-c t)]$ and have made the Bousinessq approximation. In 2-5 Pr is the Prandtl number and the Reynolds number and (bulk) Richardson number of the flow are defined as

$$
R e=\frac{U_{m} h}{2 \nu}, \quad R i=\gamma g h\left(T_{1}-T_{0}\right) / 16 U_{m}^{2},
$$

where $\gamma$ is the coefficient of thermal expansion and $g$ is the acceleration due to gravity. Our prime concern will be with the case of unstably stratified flows for which $T_{1}<T_{0}$ and thus $R i<0$.
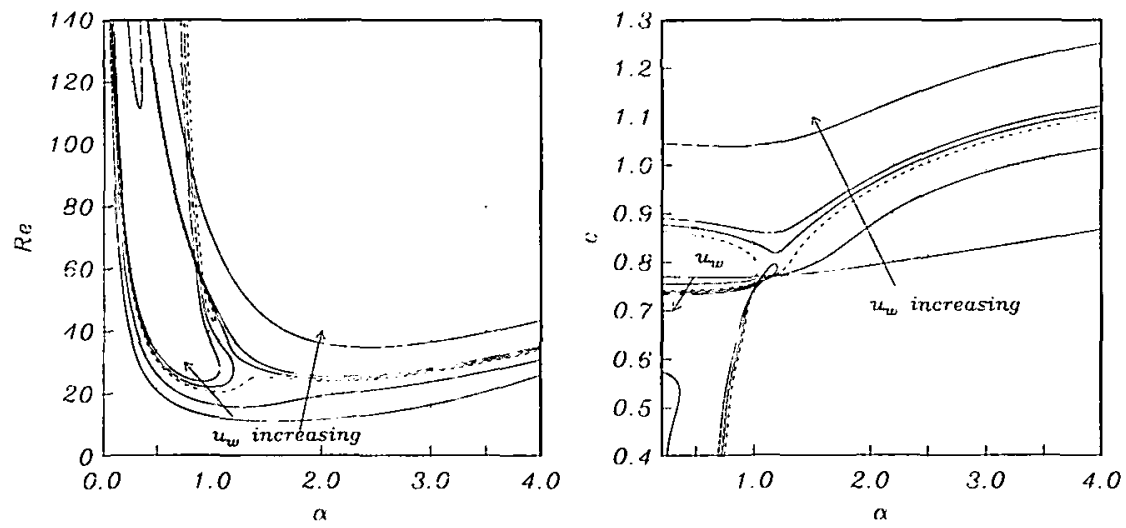

FIGURE 2. Neutral curves for $u_{w}=0.0,0.75,0.9,0.925,0.95,1.2$. Here $R i=-0.25$ and the Prandtl number $\operatorname{Pr}=1$. As a point of reference the neutral curve(s) for $u_{w}=0.9$ are drawn as dashed lines.

Before proceeding with a discussion of the high Reynolds number regime we summarize some of the stability properties of the flow at small to moderate values of the Reynolds number. To this end we present in Figure 2 a series of neutral curves in Reynolds number-wavenumber space for the eigenvalue problem 2-5; the neutral curves are for the particular case of Richardson number $R i=-0.25$ and unit Prandtl number. The lowest curve in Figure 2a corresponds to simple heated Poiseuille flow $\left(u_{w}=0\right)$. From this figure we can easily describe the effect of basic shear, here measured in terms of the parameter $u_{w}$, on the stability characteristics of the flow. Increasing $u_{w}$ from zero is seen to increase the critical Reynolds number of the flow.

Figures $2 \mathrm{a}, \mathrm{b}$ also demonstrate that it is no simple matter to describe succinctly the effect of basic shear on the critical wavenumber or the critical wavespeed of the 

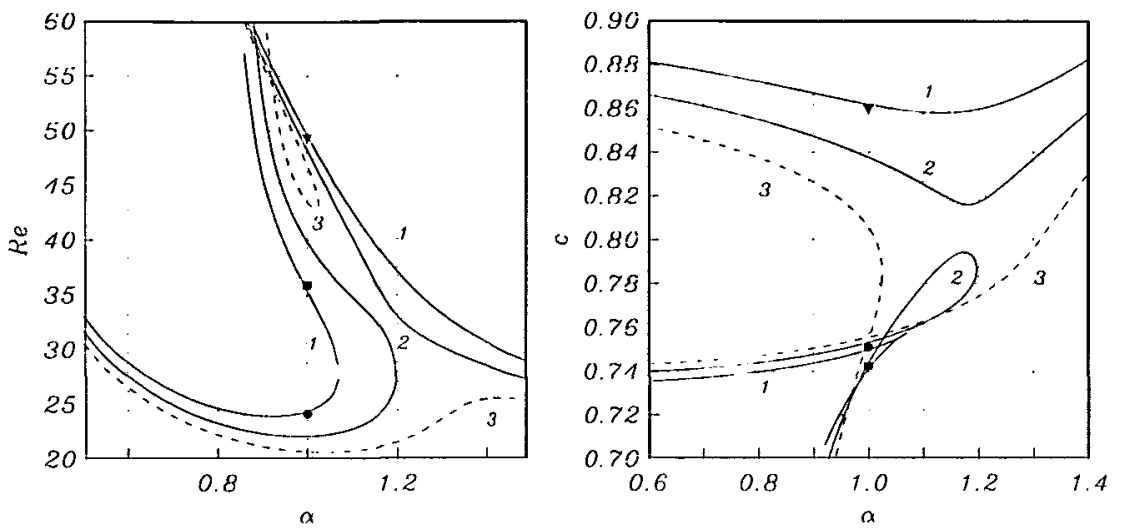

FIGURE 3. An expanded view of Figure 2. Curves labeled according to $\boldsymbol{u}_{w}=0.95$ (curve 1), $u_{w}=0.925$ (curve 2) and $u_{w}=0.9$ (curve 3).

flow. Figure 2a indicates that there is a threshold value of the shear $u_{w}$ beyond which the typical single neutral curve (with a well defined left and right hand branch as is exhibited by the $u_{w}=0$ case) which occurs for stratified Poiseuille flow splits into two distinct neutral curves. Figure 3 presents an expanded view of Figure 2 from which we can readily see the effect of increasing $u_{w}$. At a value of $u_{w}=0.9$ (the curve labelled 3) a single neutral curve exists with a secondary node or loop appearing at a higher value of the Reynolds number. For larger $u_{w}$ these two nodes of neutral curves approach and ultimately exchange identities at a critical value of the shear $u_{w}$. As $u_{w}$ is further increased the, now distinct, neutral curves move progressively further apart (see curve 2 for which $u_{w}=0.925$ and curve 1 for which $u_{w}=0.95$ ). In Figure 4 we present plots of the eigenfunctions corresponding to those points marked with symbols on curve 1 of Figure 3 (with parameter values $u_{w}=0.95$ and $\alpha=1.0$ ). From this figure we note that there is no obvious way in which to uniquely classify these modes (for example as mode 1 or mode 2 depending on the number of turning points that the eigenfunction possesses). For definiteness we will designate those eigen-modes lying along the right branch of curve 1 in Figure $3 \mathrm{a}$ as first modes and those lying along the left branch of curve 1 as second modes.

Returning to Figure 2 we note that, as we increase the level of shear $u_{w}$, the neutral curves split (through the interaction between the two modal solutions; see Figure 3) with the critical Reynolds number being associated with the first mode. As we further increase $u_{w}$ the second mode is significantly stabilized (corresponding to a dramatic increase in the critical Reynolds number) whereas the critical Reynolds number for the first mode, which resides along the right-hand lobe of the neutral curves, changes slowly. Thus, for example, the two uppermost neutral curves in Figure 2 (which correspond to a value of $u_{w}=1.2$ ) have local minima of $R e \approx 34.85$ and $\operatorname{Re} \approx 111.2$ 

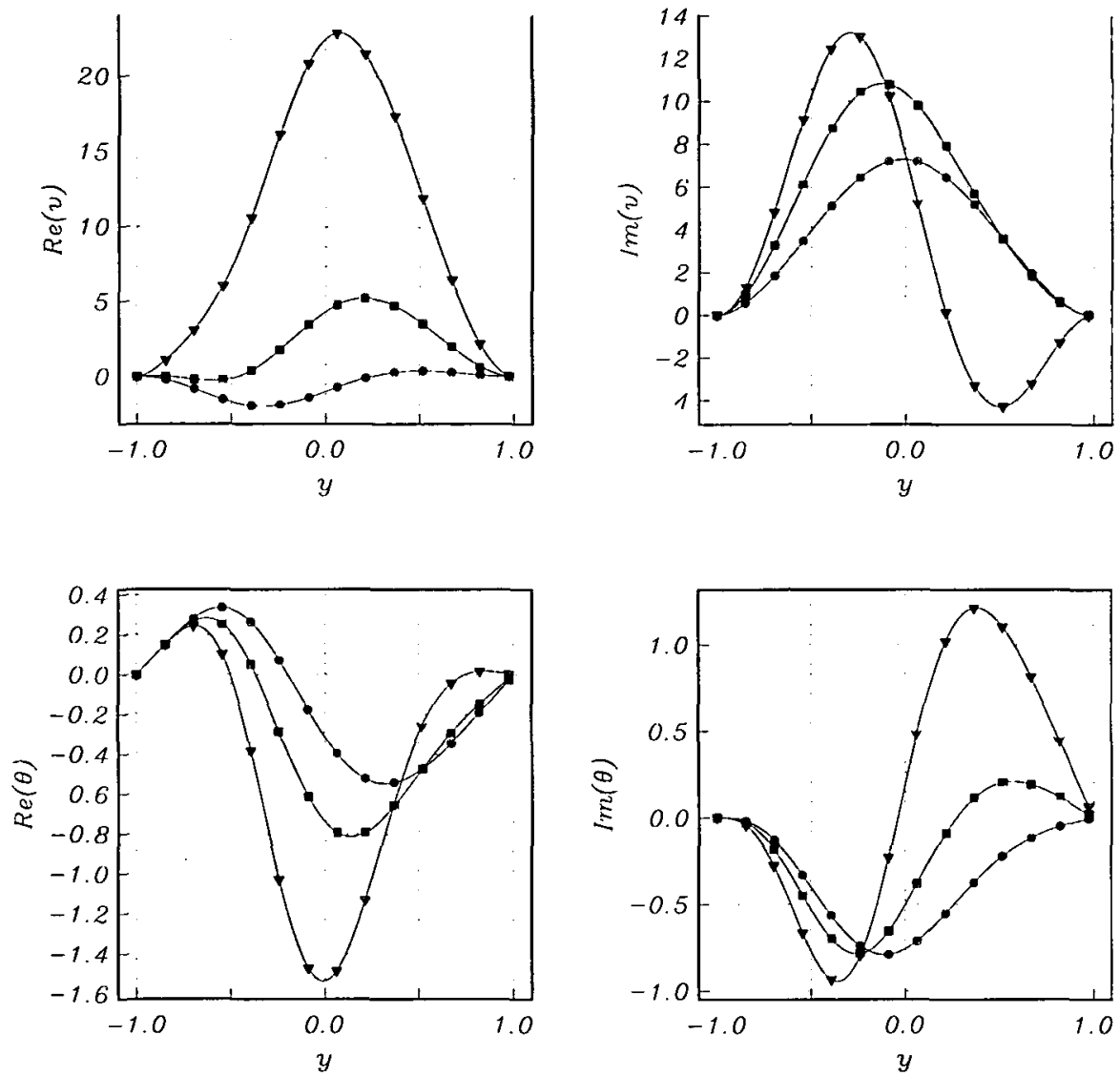

FIGURE 4. The eigenfunctions of the system (2)-(5) corresponding to those points labelled with symbols in Figure 3.

at wavenumbers of $\alpha \approx 2.4$ and $\alpha \approx 0.342$ respectively.

In consequence, we deduce that for increasing shear $u_{w}$ the critical Reynolds number and the critical wavenumber are shifted to progressively higher levels and it is the first mode of instability which dominates the flow. At these higher values of Reynolds and wave-numbers the numerical solution of 2-5 becomes increasingly difficult. Hence, in order to explore the ultimate re-stabilization of the flow as $u_{w}$ is increased we must focus our attention on the large Reynolds number and large wave-number asymptotic regimes. In Section 3 we will consider the asymptotic limit $\operatorname{Re} \rightarrow \infty$ with $\alpha=O(1)$, corresponding to inviscid wave motions, and in Section 4 examine the further limit of $\alpha \rightarrow \infty$. This will then allow us to describe the eventual restabilization of the flow at high levels of wall shear. 


\section{Inviscid Wave Motions}

The equation governing inviscid disturbances to the basic flow 1 is obtained by formally taking the limit $R e \rightarrow \infty$ in the governing equations $2-5$ whilst holding the wave number $\alpha$ and wave speed $c$ as $O(1)$ quantities. Upon eliminating the pressure, temperature and streamwise velocity component from the resulting equations we obtain the classical Taylor-Goldstein form

$$
(\bar{u}-c)^{2}\left(\frac{\partial^{2}}{\partial y^{2}}-\alpha^{2}\right) v-\left[(\bar{u}-c) \bar{u}_{y y}-R i \bar{T}_{y}\right] v=0,
$$

which must be solved subject to the boundary conditions

$$
v=0 \quad \text { on } \quad y=-1,1 .
$$

The literature on the Taylor-Goldstein equation is vast and the reader is referred to [7] for an in-depth discussion of the properties of its solutions. Of predominant concern here is with the structure of the unstable modal solutions of 6 . In Figure 5 we present a plot of the growth rates of the first mode as a function of the wavenumber for various values of the wall shear $u_{w}$; the Richardson number has been set to $R i=-1$. (The effect of varying the Richardson number can be summarised succinctly as (i): increasing the magnitude of the negative Richardson number increases the magnitude of the growth rate and (ii): unstable modes can only exist for $R i<0$.)
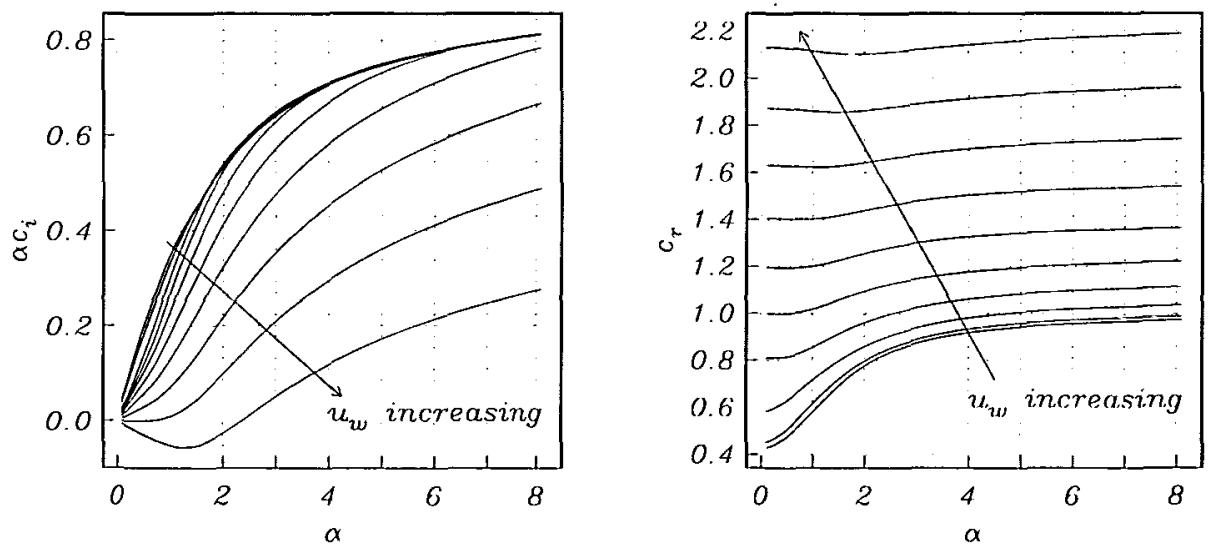

FIGURE 5. Plot of the growth rate and wavespeed of the first eigen-mode of equation (6) as a function of wavenumber $\alpha$ for various values of $u_{w}=0.0,0.25, \ldots, 2.25$.

Several features are readily discerned from Figure 5. First, the flow is unstable to modes of $O(1)$ wavelength and the magnitude of the growth rate decreases as the value 
of the wall shear $u_{w}$ is increased (for a fixed value of the Richardson number). The second noticeable feature is that as $u_{w}$ is increased it is the relatively long wavelength motions which are first rendered stable. This is in perfect agreement with our results of section 2 where, from Figure 2 we note that the neutral curve for mode 1 moves to progressively higher values of the Reynolds and wave-numbers as the level of shear $u_{w}$ is increased.

In the case of neutral wave modes, with wavespeed $c_{r}$, equation 6 is singular at the critical point $y_{c}$ where $\bar{u}\left(y_{c}\right)=c_{r}$. Thus in order to determine the values of the neutral wavenumber and wavespeed equation 6 must be integrated along a suitably indented contour in the complex plane. Standard critical layer theory (see the references below) shows that as long as our chosen contour passes from $y=-1$ to $y=1$ in the complex $y$-plane such that it lies above $y=y_{c}$ then straightforward numerical techniques will recover the desired neutral wavespeed. The advantage of using a deformed contour which passes well away from the critical point is that on the contour 6 is nowhere singular and hence can be solved subject to 7 without the need to appeal to elegant numerical procedures. In the usual way we first took advantage of the linearity of this equation and normalized $v$ such that $v_{y}=1$ on $y=-1$. Equation 6 with boundary conditions $v=0$ and $v_{y}=1$ on $y=-1$ was integrated 6 in the complex plane along the indented contour $y+i\left(1-y^{2}\right)$ (where $\left.y \in[-1,1]\right)$ using a fourth order Runge-Kutta scheme. Finally Newton iteration was applied to the estimates of neutral wavenumber and wavespeed until the boundary condition $v=0$ on $y=1$ was satisfied to within some desired tolerance.
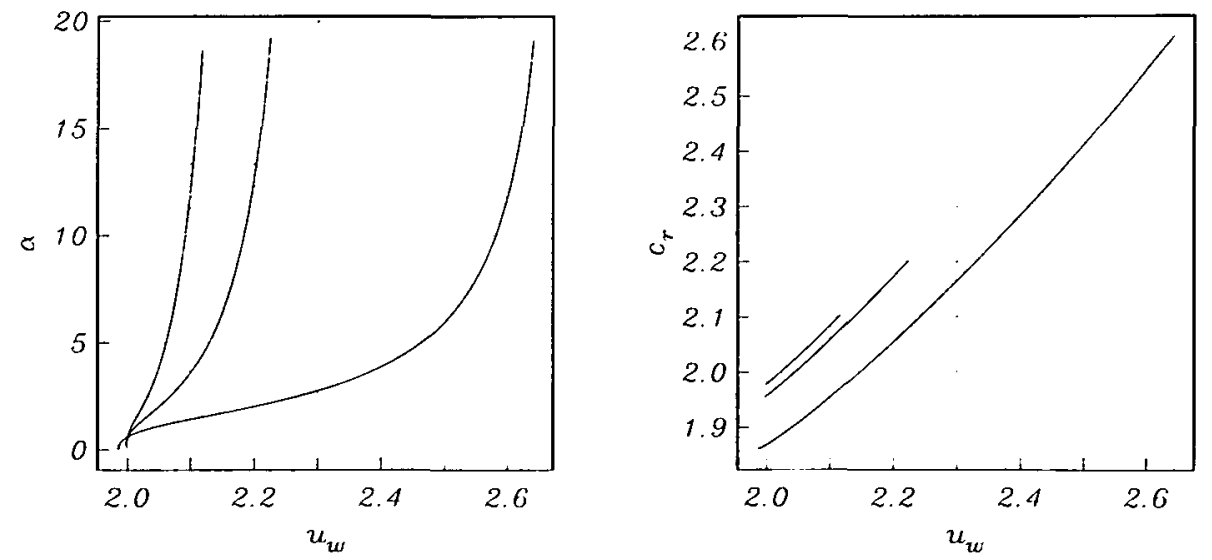

FIGURE 6. Plot of the neutral wavenumber and wave speed versus $u_{w}$ for the first three eigen-modes of equation (6).

A plot in Figure 6 of the neutral wavenumber and wavespeed for the first three modal 
solutions of 6 indicates that as $u_{w}$ is increased so the neutral wavenumbers grow and there is a value of shear beyond which the flow is stable to inviscid disturbances (that is, the neutral wavenumber becomes unbounded at a finite value of $u_{w}$ ). In order to complete the description of the neutrally stable inviscid wave modes we must, of course, consider the behaviour in the critical layer located at the position $y_{c}$ where $\bar{u}\left(y_{c}\right)=c_{r}$. The critical layer serves to smooth, through the re-introduction of viscosity at the critical level, the singularity encountered in the inviscid equation. However as this analysis is now standard, and the results well known, we refer the interested reader to Miles [15] or Drazin \& Reid [7] for a comprehensive discussion of the behaviour of the critical layer of the Taylor-Goldstein equation in a stratified fluid. Further, a recent paper by Blackaby \& Choudhari [2] provides an interesting account of the Taylor-Goldstein equation, its numerical solution and its importance to the stability of weakly three-dimensional boundary layer flows. Thus by increasing the level of wall shear $u_{w}$ the instability is driven to progressively shorter wavelengths and in order to describe accurately the eventual restabilization of the flow we must focus our attention on the combined large Reynolds number-wave number asymptotic regime.

Turning our attention back to Figure 5 we remark that in the small wavelength limit both the growth rate $\alpha c_{i}$ and the wavespeed $c_{r}$ tend to constant limiting values whose magnitudes decrease, in the case of $\alpha c_{i}$, and increase, in the case of $c_{r}$, as $u_{w}$ is increased. This phenomena is precisely that described by Mureithi et al. [16] who were concerned with the stability of mixed free-forced convection boundary layers. As such we can anticipate from the results of [16] that in the limit $\alpha \rightarrow \infty$ the wave modes become increasingly localized about the position where the streamwise velocity attains its maximum value. Owing to this spatial localization of the wave mode viscous effects serve to render the flow stable at some sufficiently large value of the streamwise wavenumber. In terms of the wall shear $u_{w}$ the position of maximum streamwise velocity is given by $y_{\max }=u_{w} / 2$ and as such the position of maximum velocity is attained at the upper boundary when $u_{w}=2$. (Note also that the maximum velocity is attained at the lower boundary if $u_{w}=-2$; as only minor modifications are required to the analysis to be presented for the case $u_{w}>0$ in order to consider the case of $u_{w}<0$ we will restrict our attention to the regime $u_{w} \geq 0$.) Thus as the level of wall shear approaches a value at which the maximum streamwise velocity occurs at the upper boundary the results of [16] must be modified in order to account for the presence of the rigid boundary.

We now turn our attention to the combined asymptotic limits of large Reynolds number and large wave number in order to determine the level of wall shear at which the flow becomes stabilized. 


\section{The Right-Hand Branch of the Neutral Curve}

Our examination of the right-hand branch behaviour begins with the case for which the shear $0<u_{w}<2$ so that the maximum streamwise velocity occurs away from the bounding plates. The analysis is then similar to that of Mureithi et al. [16] but is necessary in order to anticipate the important details of the flow structure relevant to $u_{w} \geq 2$ when the maximum flow speed is located on the upper plate - a problem which is considered in Sections 4.2 and 4.3.

4.1. The case $0<u_{w}<2$. To determine the dominant spatial and temporal scales we first note that in order to achieve a balance between streamwise diffusion, as represented by the term $-\alpha^{2} v$ in the vertical momentum equation 3 , and thermal buoyancy, corresponding to the term $-4 R i R e \theta$ in 3 , in the combined large Reynolds number-wave number limit we must choose $\alpha=O\left(R e^{1 / 2}\right)$ and $v=O(\theta)$. Secondly from Figure 5 we note that in the large wavenumber limit the inviscid wavespeed $c_{r}$ tends to a constant thus suggesting that in the combined limit of $\operatorname{Re} \rightarrow \infty$ and $\alpha \rightarrow \infty$ we must choose $c=O(1)$. (As we are concerned with neutral wave modes $c$ will be taken to be real throughout this and subsequent sections.)

In anticipation of the result that the neutral wave modes travel at the maximum speed of the flow we may determine the appropriate normal length-scale of the righthand branch modes by writing $z=\operatorname{Re}^{n}\left(y-y_{0}\right)$ where $y_{0}$ is the position at which the basic flow attains its maximum velocity. On expanding

$$
(\bar{u}-c)=\left(\bar{u}\left(y_{0}\right)-c_{0}\right)+\frac{1}{2} \bar{u}^{\prime \prime}\left(y_{0}\right) R e^{-2 n} z^{2}+\cdots
$$

we obtain a balance between normal diffusion and streamwise advection when

$$
\operatorname{Re}^{2 n} \frac{\partial^{2}}{\partial z^{2}} \sim \alpha \operatorname{Re}^{1-2 n} \bar{u}^{\prime \prime}\left(y_{0}\right) z^{2}
$$

which, recalling that $\alpha=O\left(R e^{1 / 2}\right)$, gives the result that $n=3 / 8$. Thus the neutral right-hand branch wave modes will be confined within a viscous layer of thickness $O\left(\operatorname{Re}^{-3 / 8}\right)$ centred on the location $y_{0}$.

To proceed we define $z=R e^{3 / 8}\left(y-y_{0}\right)$ and expand

$$
\alpha=\operatorname{Re}^{1 / 2}\left(\alpha_{0}+\operatorname{Re}^{-1 / 4} \alpha_{1}+\cdots\right), \quad c=c_{0}+\operatorname{Re}^{-3 / 4} c_{1}+\cdots,
$$

where we anticipate the result that $c_{0}=\bar{u}\left(y_{0}\right)$ (so that the neutral wave-modes propagate downstream with a speed equal to the maximum value of the streamwise velocity). Furthermore, we expand the velocity, temperature and pressure fields as

$$
\begin{aligned}
(u, v, \theta, p)= & \left(R e^{-1 / 8} U_{0}, V_{0}, \Theta_{0}, R e^{-5 / 8} P_{0}\right) \\
& +R e^{-1 / 4}\left(R e^{-1 / 8} U_{1}, V_{1}, \Theta_{1}, R e^{-5 / 8} P_{1}\right)+\cdots .
\end{aligned}
$$


Note that with the magnitude of the vertical velocity component chosen to be $O(1)$ the temperature disturbance $\theta$ must, in light of our discussion above, also be an $O(1)$ quantity. The relative scalings for $u$ and $p$ in 8 then follow from a dominant balance in the continuity and streamwise momentum equations.

Substitution of 8 into the governing equations 2-5 and equating coefficients of inverse powers of $\operatorname{Re}^{1 / 4}$ to zero gives, at leading order

$$
\begin{aligned}
\alpha_{0}^{2} U_{0}+i \alpha_{0} P_{0}=0, & i \alpha_{0} U_{0}+V_{0 z}=0, \\
\alpha_{0}^{2} V_{0}-4 R i \Theta_{0}=0, & \alpha_{0}^{2} \Theta_{0}+\operatorname{Pr} V_{0}=0 .
\end{aligned}
$$

Equations 9 and 10 are compatible (that is, yield a non-trivial solution) if

$$
\alpha_{0}^{4}=-4 \operatorname{Pr} R
$$

so that the neutrally stable wave modes to be described here can only exist for Richardson numbers $R i<0$ with the neutral wavenumber given by 11 . At next order in our expansion we obtain an inhomogeneous version of equations 9-10 for the quantities $U_{1}, V_{1}$ etc which are compatible provided

$$
3 \frac{\partial^{2} V_{0}}{\partial z^{2}}+\left\{i \alpha_{0}(1+\operatorname{Pr})\left(z^{2}+c_{1}\right)-4 \alpha_{0} \alpha_{1}\right\} V_{0}=0
$$

which must be solved subject to the constraint that $V_{0} \rightarrow 0$ as $|z| \rightarrow \infty$ (in order to ensure the wave mode is confined to the viscous layer centred on $y_{0}$ ). Thus, equation 12 defines an eigenvalue problem for the first order correction to the neutral wavenumber $\alpha_{1}$ and wave speed $c_{1}$. This equation can be solved in terms of Parabolic Cylinder functions (see [1]) from which we can deduce readily that in order to satisfy the required far field boundary conditions we require

$$
\alpha_{1}=-\frac{2 n+1}{4} \sqrt{\frac{3(1+P r)}{2 \alpha_{0}}}, \quad c_{1}=-\frac{2 n+1}{4(1+P r)} \sqrt{\frac{3(1+P r)}{2 \alpha_{0}}}, \quad n=0,1,2, \ldots
$$

Thus the neutral curve corresponding to the most unstable mode (i.e. $n=0$ ) is given by

$$
\alpha=\alpha_{0} \operatorname{Re}^{1 / 2}\left(1-\frac{1}{4} \sqrt{\frac{3(1+P r)}{2 \alpha_{0}^{3}}} \operatorname{Re}^{-1 / 4}+\ldots\right),
$$

where $\alpha_{0}$ is given by the positive root of equation 11 .

4.2. The case $u_{w}=2+O\left(\operatorname{Re}^{-3 / 8}\right)$. The structure described in the previous section is only valid provided the position of maximum streamwise velocity $y_{0}$ is not located at the upper rigid boundary. Thus, as $y_{0} \rightarrow 1$, corresponding to $u_{w} \rightarrow 2$, a new 
structure must emerge. We now consider the modifications required to our previous analysis in order to describe the neutral wave modes.

It is a simple matter to show that the structure described in Section 4.1 is first significantly modified when $u_{w}=2+O\left(R e^{-3 / 8}\right)$. Thus, let us write

$$
u_{w}=2+\operatorname{Re}^{-3 / 8} \tilde{u}_{w}^{*}
$$

where $\tilde{u}_{w}^{*}$ is an $O(1)$ quantity (which can be positive or negative). We again define a new stretched variable $z=R e^{3 / 8}(y-1)$ and, in light of our result from Section 4.1 that $c_{0}=\bar{u}\left(y_{0}\right)$, expand the wavenumber and wavespeed as

$$
\alpha=\operatorname{Re}^{1 / 2}\left(\alpha_{0}+\operatorname{Re}^{-1 / 4} \alpha_{10}+\cdots\right), \quad c=2+\operatorname{Re}^{-3 / 8} \tilde{u}_{w}^{*}+\operatorname{Re}^{-3 / 4} c_{10}+\cdots .
$$

Expanding the dependent variables as power series in inverse powers of $R e^{1 / 4}$ we again find that the leading order neutral wavenumber $\alpha_{0}$ is given by 11 . At next order a solvability condition on the first order correction to the temperature and vertical velocity components gives that $V_{0}$ (the leading order component of the vertical velocity) satisfies:

$$
3 \frac{\partial^{2} V_{0}}{\partial z^{2}}-\left\{i \alpha_{0}(1+\operatorname{Pr})\left(\tilde{u}_{w}^{*} z-z^{2}-c_{10}\right)+4 \alpha_{0} \alpha_{10}\right\} V_{0}=0
$$

this must be solved subject to the constraint that $V_{0} \rightarrow 0$ as $z \rightarrow-\infty$ and the usual inviscid boundary condition $V_{0}=0$ on $z=0$.

Equation 15 can be scaled to a canonical form

$$
\frac{\partial^{2} V_{0}}{\partial \phi^{2}}-\left(\Omega-i \Delta \phi-2 i \phi^{2}\right) V_{0}=0
$$

with boundary conditions

$$
V_{0}=0 \quad \phi=0, \infty,
$$

by defining $z=-\mu \phi$ and

$$
\Omega=-\frac{1}{3} i \alpha_{0}(1+P r) \mu^{2} c_{10}+\frac{4}{3} \alpha_{0} \alpha_{10} \mu^{2}, \quad \Delta=\frac{1}{3} \alpha_{0}(1+\operatorname{Pr}) \mu^{3} \tilde{u}_{w}^{*},
$$

where $\mu^{4}=6 /\left[\alpha_{0}(1+P r)\right]$. Although this equation can be solved in terms of Parabolic Cylinder functions the resulting eigenrelation, which determines the leading order correction to the wavenumber $\alpha_{10}$ and wavespeed $c_{10}$ as functions of $\tilde{u}_{w}^{*}$, is not in a form which is suitable for computational purposes. We chose instead to pose 16 as a two point boundary value problem and solve for $\Omega$ as a function of $\Delta$; this was done using a second order finite difference discretization of 16 coupled with a Newton iteration procedure to determine $\Omega$. We present the results of our calculation 

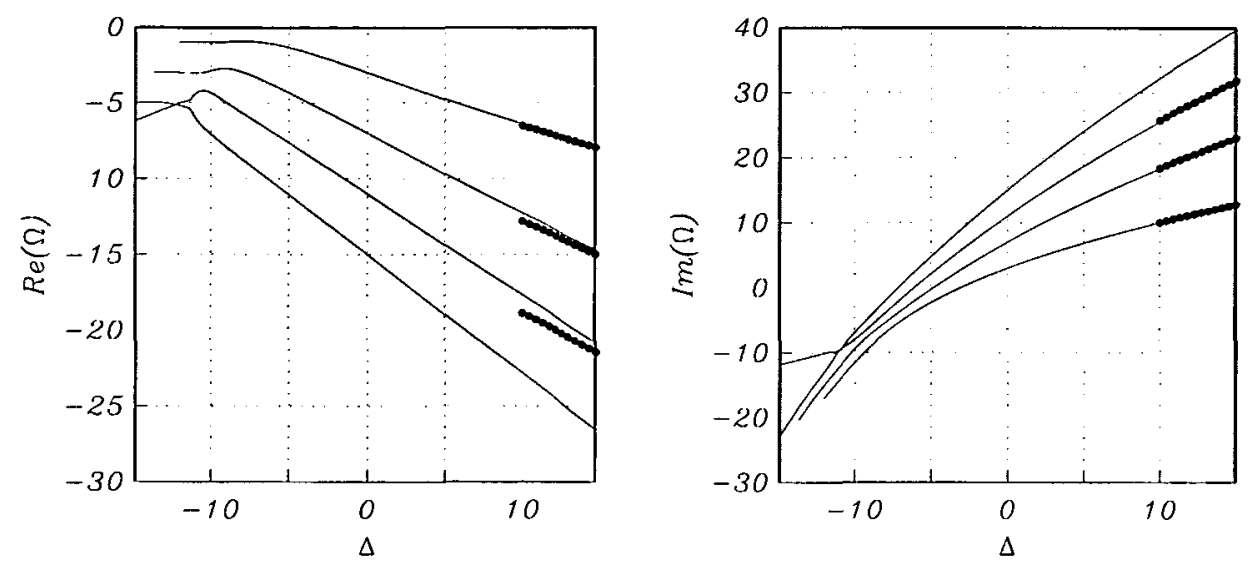

FIGURE 7. Plot of the first four eigenvalues of equation (16). The large $\Delta$ asymptotes (19) are marked for the first three modes.

in Figure 7. As a simple check on the accuracy of our numerical scheme, we note that when $\tilde{u}_{w}^{*}=0$ (and so $\Delta=0$ in Figure 7) exact values for $\Omega$ may be obtained from analytic solution of 16 ; these are $\Omega=-(2 n+1)(1-i),(n=0,1,2, \ldots)$ which are in perfect agreement with our numerical results in Figure 7.

We observe from Figure 7 that in the limit $\tilde{u}_{w}^{*} \rightarrow-\infty$ we have $\operatorname{Re}(\Omega) \rightarrow$ constant. The permissible values of this limiting constant are $-1,-3,-5, \cdots$ and, on appeal to 17 , we deduce that

$$
\alpha_{10} \rightarrow-\frac{(2 n+1)}{4} \sqrt{\frac{3(1+P r)}{2 \alpha_{0}}} \quad \text { as } \quad \tilde{u}_{w}^{*} \rightarrow-\infty ;
$$

these are the values of $\alpha_{1}$ predicted from the analysis in Section 4.1 (see equation 13). The eigenfunctions corresponding to the first two modes are shown in Figure 8. Here we see that the large negative $\Delta=-10.0$ eigenfunctions (the dashed curves in Figure 8) are concentrated away from the boundary and so in the limit $\tilde{u}_{w}^{*} \rightarrow-\infty$ the solutions of 15 match directly to the solutions of Section 4.1.

In Figure 9a we present a plot of the third eigen-mode solution of 16. From this figure we observe that there is an additional asymptotic solution in the limit $\Delta \rightarrow-\infty$ for which $\operatorname{Re}(\Omega)$ and $\operatorname{Im}(\Omega)$ both tend to $-\infty$. The corresponding eigenfunctions for this secondary mode are presented in Figure $9 \mathrm{~b}$ from which we see that in the limit $\Delta \rightarrow-\infty$ the eigen-modes become localized at the upper boundary. Writing $\Delta=-\Gamma$, where $\Gamma \gg 1$, and defining $\phi=\Gamma^{-1 / 3} r, \Omega=\Gamma^{2 / 3} \tilde{\Omega}$ we obtain from 16 , at leading order,

$$
\frac{\partial^{2} V_{0}}{\partial r^{2}}-(i r+\tilde{\Omega}) V_{0}=0
$$



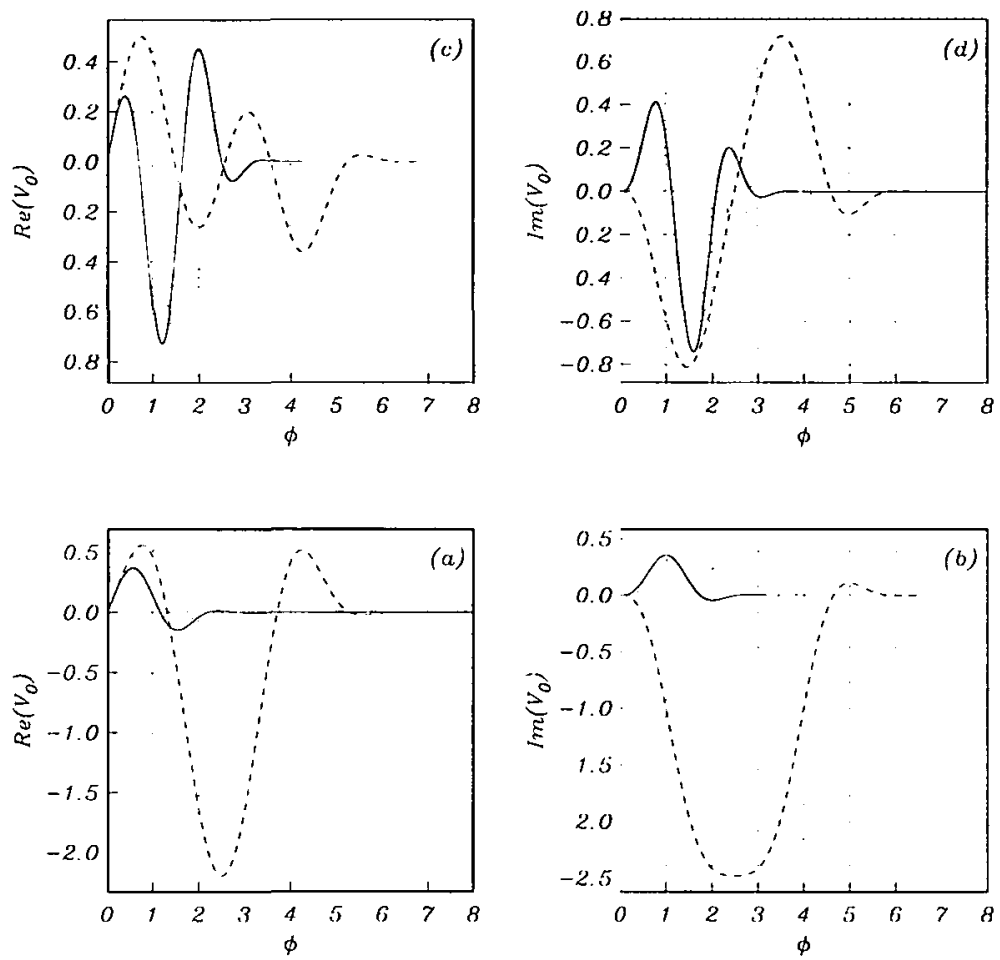

FIGURE 8. Plot of the first two eigenfunctions of equation (16). Shown are results for $\Delta=-10.0$ (dashed curve), $\Delta=0$ (dotted curve) and $\Delta=10.0$ (solidus). (a)-(b) Mode 1, (c)-(d) Mode 2.

which has the bounded solution

$$
V=\mathrm{Ai}\left[e^{i \pi / 6}(r-i \tilde{\Omega})\right],
$$

where Ai is the usual Airy function (see Abramowitz \& Stegun [1]). In order for this solution to vanish at $r=0$ we require $\mathrm{Ai}\left[e^{-i \pi / 3} \tilde{\Omega}\right]=0$. The first zero of the Airy function is given by $\tilde{\Omega}=-2.3381 e^{i \pi / 3}$ and so for $\Gamma \gg 1$ we have

$$
\operatorname{Re}(\Omega) \approx-1.169 \Gamma^{2 / 3}+\cdots, \quad \operatorname{Im}(\Omega)=-1.169 \sqrt{3} \Gamma^{2 / 3}+\cdots .
$$

The asymptotes 18 are in excellent agreement with the numerical solutions given in Figure 9 and the difference between the asymptotic and numerical solutions for $\Delta<-5$ is graphically almost undetectable. There are, of course, an infinite number of wall bounded modes - one corresponding to each zero of the Airy function $\mathrm{Ai}$ - but it becomes increasingly difficult to determine numerically these higher modes. This is not a serious deficiency since the dominant mode in the flow is the viscous one 

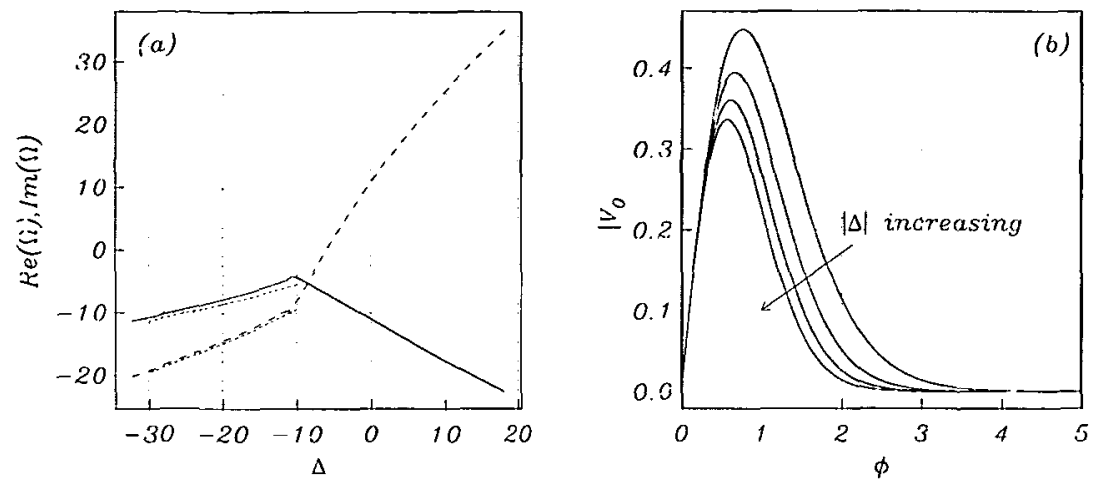

FIGURE 9. Plot of the third eigen-mode of equation (16). Shown are (a) $\operatorname{Re}(\Omega)$ (solidus), $\operatorname{Im}(\Omega)$ (dashed) versus $\Delta$ and the asymptotic limit given by (18) (dotted) and (b) $\left|V_{0}\right|$ versus $\phi$ for $\Delta=$ $-15,-20,-25,-30$.

localized about the position of maximum streamwise velocity. The secondary mode is thus relegated to a position of mere academic interest with regards to the overall stability characteristics of the flow in the limit $\Delta \rightarrow-\infty$.

The second asymptotic limit which arises from Figure 7 is $\tilde{u}_{w}^{*} \rightarrow \infty$. Here we note that $\alpha_{10} \rightarrow-\infty$ and $c_{10} \rightarrow-\infty$ as $\tilde{u}_{w}^{*} \rightarrow \infty$ and Figure 8 demonstrates that the modes become increasingly localized to the vicinity of the upper boundary $z=0$.

In order to examine the case $\Delta \rightarrow \infty$ in 16 (corresponding to $\tilde{u}_{w}^{*} \rightarrow \infty$ ) let us first define $\phi=\Delta^{-1 / 3} q$ and write $\Omega=\hat{\Omega}_{0} \Delta^{2 / 3}+\hat{\Omega}_{1} \Delta^{-2 / 3}+\cdots$. At leading order equation 16 becomes

$$
\frac{\partial^{2} V_{0}}{\partial q^{2}}+\left(i q-\hat{\Omega}_{0}\right) V_{0}=0
$$

which has the solution

$$
V_{0}=\mathrm{Ai}\left[e^{-i \pi / 6}\left(q+i \hat{\Omega}_{0}\right)\right]
$$

which automatically satisfies the requirement that $V_{0} \rightarrow 0$ as $q \rightarrow \infty$. In order to vanish at $q=0$ we require

$$
\operatorname{Ai}\left(e^{i \pi / 3} \hat{\Omega}_{0}\right)=0
$$

and therefore

$$
\hat{\Omega}_{0}=e^{-i \pi / 3} s_{0},
$$

where $s_{0}$ corresponds to the zero(s) of the Airy function; the first four are given by

$$
s_{0}=(-2.3381,-4.0879,-5.5206,-6.7867, \cdots) .
$$

At next order we obtain an inhomogeneous version of the Airy equation, which when solved subject to the conditions that its solution vanishes at $q=0$ and as $q \rightarrow \infty$ 
yields

$$
\hat{\Omega}_{1}=-\frac{16}{15} s_{0}^{2} e^{-i \pi / 6} .
$$

Thus

$$
\Omega \approx \Delta^{2 / 3} s_{0} e^{-i \pi / 3}-\frac{16}{15} s_{0}^{2} \Delta^{-2 / 3} e^{-i \pi / 6}+\cdots
$$

For comparison the first three asymptotes $\Omega=\hat{\Omega}_{0} \Delta^{2 / 3}+\hat{\Omega}_{1} \Delta^{-2 / 3}+\ldots$ as $\Delta \rightarrow \infty$ are shown in Figure 7. Excellent agreement with the numerical results is evident even at moderate values of the parameter $\Delta$.

The solutions described above do not satisfy the full no-slip boundary conditions which must be imposed at the upper rigid boundary $y=1$. This deficiency is remedied by the re-introduction of viscosity into the problem in the form of a viscous layer of thickness $O\left(R e^{-1 / 2}\right)$. However as this viscous layer is passive we do not present details of it here; the interested reader is referred to the paper by Hall [12] where a similar layer is encountered in the study of short wavelength Taylor vortices in the flow between rotating concentric cylinders.

4.3. The case $u_{w}=2+O(1)$. The previous sections demonstrate how the neutral wave structure is first modified as $u_{w} \rightarrow 2$. As $u_{w}$ is increased past 2 the position at which the neutral wave mode is localized occurs on the rigid boundary $y=1$. However, the leading order neutral wavenumber remains unaffected whilst $u_{w}=$ $2+o(1)$. We now turn our attention to the final distinguished limit at which the leading order wavenumber is modified by the large level of wall shear.

If we write $\tilde{u}_{w}^{*}=O\left(R e^{n}\right)$, see 14 , so that $u_{w}=2+O\left(R e^{n-3 / 8}\right)$, we have, as a result of the large $\Delta$ analysis presented above,

$$
\alpha=\operatorname{Re}^{1 / 2}\left(\alpha_{0}+O\left(R e^{2 n / 3-1 / 4}\right)\right),
$$

and the wave mode is restricted to an $O\left(R e^{-n / 3-3 / 8}\right)$ depth viscous layer situated at the boundary $y=1$. This wavenumber expansion becomes disordered when $n=3 / 8$ in which case $u_{w}=2+O(1)$ and the wave mode is confined to an $O\left(R e^{-1 / 2}\right)$ viscous layer located at $y=1$. On this basis we write

$$
u_{w}=2+u_{w 0}, \quad \alpha=R e^{1 / 2} \alpha_{00}+\cdots, \quad c=2+u_{w 0}+R e^{-1 / 2} c_{11}+\cdots
$$

and define a new scaled normal variable $\tilde{z}=\operatorname{Re}^{1 / 2}(y-1)$. Furthermore we expand the velocity, temperature and pressure fields as

$$
(u, v, \theta, p)=\left(U_{0}, V_{0}, \Theta_{0}, R e^{-1 / 2} P_{0}\right)+\cdots
$$


and substitute these in the governing equations to yield, at leading order in powers of $R e^{-1 / 4}$,

$$
\begin{aligned}
\left\{\frac{d^{2}}{d \tilde{z}^{2}}-\alpha_{\infty 0}^{2}-i \alpha_{00} \operatorname{Pr}\left(u_{w 0} \tilde{z}-c_{11}\right)\right\} \Theta_{0} & =\operatorname{Pr} V_{0}, \\
\left\{\frac{d^{2}}{d \tilde{z}^{2}}-\alpha_{00}^{2}-i \alpha_{00}\left(u_{w 0} \tilde{z}-c_{11}\right)\right\} U_{0} & =i \alpha_{00} P_{0}+u_{w 0} V_{0}, \\
\left\{\frac{d^{2}}{d \tilde{z}^{2}}-\alpha_{00}^{2}-i \alpha_{00}\left(u_{w 0} \tilde{z}-c_{11}\right)\right\} V_{0} & =\frac{d P_{0}}{d \tilde{z}}-4 R i \Theta_{0}, \\
i \alpha_{00} U_{0}+\frac{d V_{0}}{d \tilde{z}} & =0
\end{aligned}
$$

which must be solved subject to the full no-slip boundary conditions

$$
U_{0}=V_{0}=\Theta_{0}=0 \quad \tilde{z}=0, \rightarrow-\infty
$$

Eliminating $U_{0}$ and $P_{0}$ gives

$$
\begin{aligned}
\left(\frac{d^{2}}{d \tilde{z}^{2}}-\alpha_{00}^{2}-i \alpha_{00}\left(u_{w 0} \tilde{z}-c_{11}\right)\right)\left(\frac{d^{2}}{d \tilde{z}^{2}}-\alpha_{00}^{2}\right) V_{0} & =4 \alpha_{00}^{2} R i \Theta_{0}, \\
\left(\frac{d^{2}}{d \tilde{z}^{2}}-\alpha_{00}^{2}-i \alpha_{00} \operatorname{Pr}\left(u_{w 0} \tilde{z}-c_{11}\right)\right) \Theta_{0} & =\operatorname{Pr} V_{0} .
\end{aligned}
$$

For computational purposes we define $\varphi=-\alpha_{00} \tilde{z}$ so 24 and 25 can be rewritten as

$$
\begin{aligned}
\left(\frac{\partial}{\partial \varphi^{2}}-1+\frac{i}{\alpha_{00}^{2}}\left(u_{w 0} \varphi+\alpha_{00} c_{11}\right)\right)\left(\frac{d^{2}}{d \varphi^{2}}-1\right) V_{0} & =4 \frac{\operatorname{Ri} \Theta_{0}}{\alpha_{00}^{2}} \\
\left(\frac{\partial}{\partial \varphi^{2}}-1+\frac{i P r}{\alpha_{00}^{2}}\left(u_{w 0} \varphi+\alpha_{00} c_{11}\right)\right) \Theta_{0} & =\frac{\operatorname{Pr} V_{0}}{\alpha_{00}^{2}}
\end{aligned}
$$

together with the boundary conditions

$$
V_{0}=\frac{\partial V_{0}}{\partial \varphi}=\Theta_{0}=0 \quad \varphi=0, \infty
$$

This system was solved using a numerical scheme developed by Otto \& Bassom [17] to solve the eigenvalue problem for the most unstable Görtler vortex mode in a weakly three-dimensional boundary layer. It relies on a fourth order finite difference discretization of the governing equations, with the boundary condition $\Theta_{0}=0$ on $\varphi=0$ replaced by $\Theta_{0 \varphi}=1$ on $\varphi=0$, and employs a coupled Davis scheme to invert efficiently the resulting coupled tri- and penta-diagonal matrix equations. Newton iteration is carried out on the unknowns, namely $\alpha_{00}$ and $c_{11}$, until the remaining 

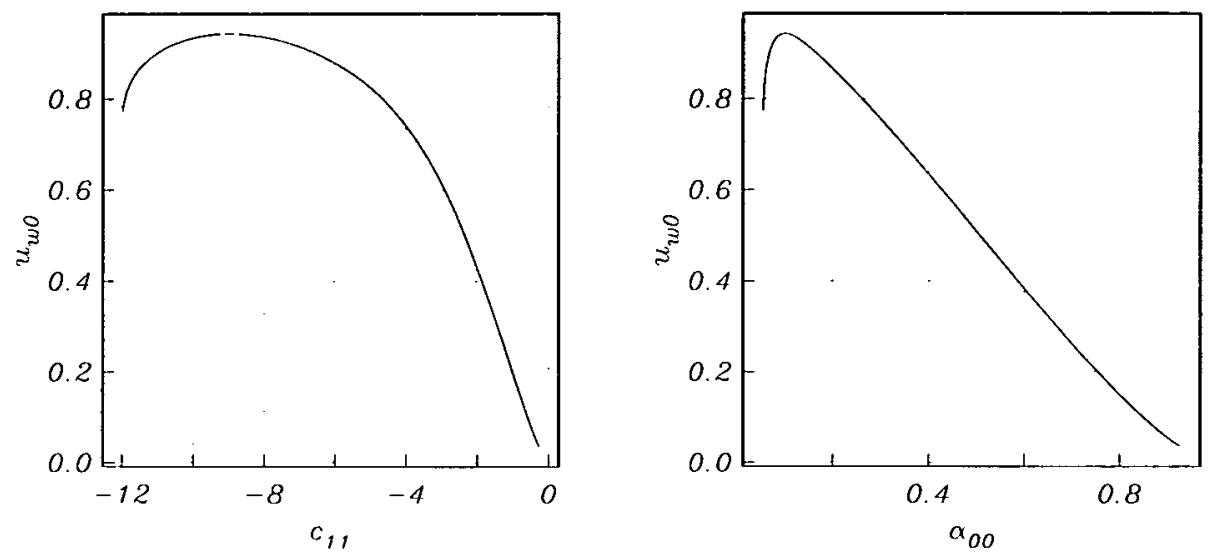

FIGURE 10. Plot of the neutral wave speed and wavenumber of the system (26)-(27) versus $u_{w 0}$. The results are for Prandtl number unity and Richardson number $R i=-1.0$. Note there is a maximum value of $u_{w 0}$ beyond which the wall layer equations can no longer support neutral wave modes.

boundary condition, $\Theta_{0}=0$ on $\varphi=0$, is satisfied (to within some desired tolerance). The reader is referred to [17] for full details.

We present the results of our calculation in Figures 10 and 11. Figure 10 shows a plot of the neutral wavenumber and wavespeed versus the wall shear correction $\boldsymbol{u}_{w 0}$. To interpret this figure we note that modes lying in the region below the $u_{w 0}$ versus $\alpha$ curve the flow are unstable. The dominant feature of this figure is that there is a maximum value of $u_{w 0} \approx 0.943164$ beyond which the flow may no longer support unstable modes. The corresponding eigenfunctions are shown in Figure 11.

In the limit $u_{w 0} \rightarrow 0$ there are two distinguished limits corresponding to the leftand right-hand branch of the neutral curve in Figure 10. Our analysis demonstrates that the right-hand branch modes match onto the viscous solutions described in Section 4 . In the small $u_{w 0}$ limit the left-hand branch modes will match directly onto the inviscid neutral solutions of Section 3 (see Figure 6). In this limit these neutral wave modes develop a critical layer structure; the reader is referred to Miles [15] or Drazin \& Reid [7] for an in-depth discussion of the critical layer behaviour. It is this development of a critical layer that makes the numerical solution of 26-27 increasingly difficult for small values of $u_{w 0}$.

\section{Conclusions}

We have considered the instability of thermally stratified Poiseuille-Couette flow to two-dimensional travelling wave motions. Particular emphasis has been placed on describing the stability characteristics of the flow as the level of shear, arising 

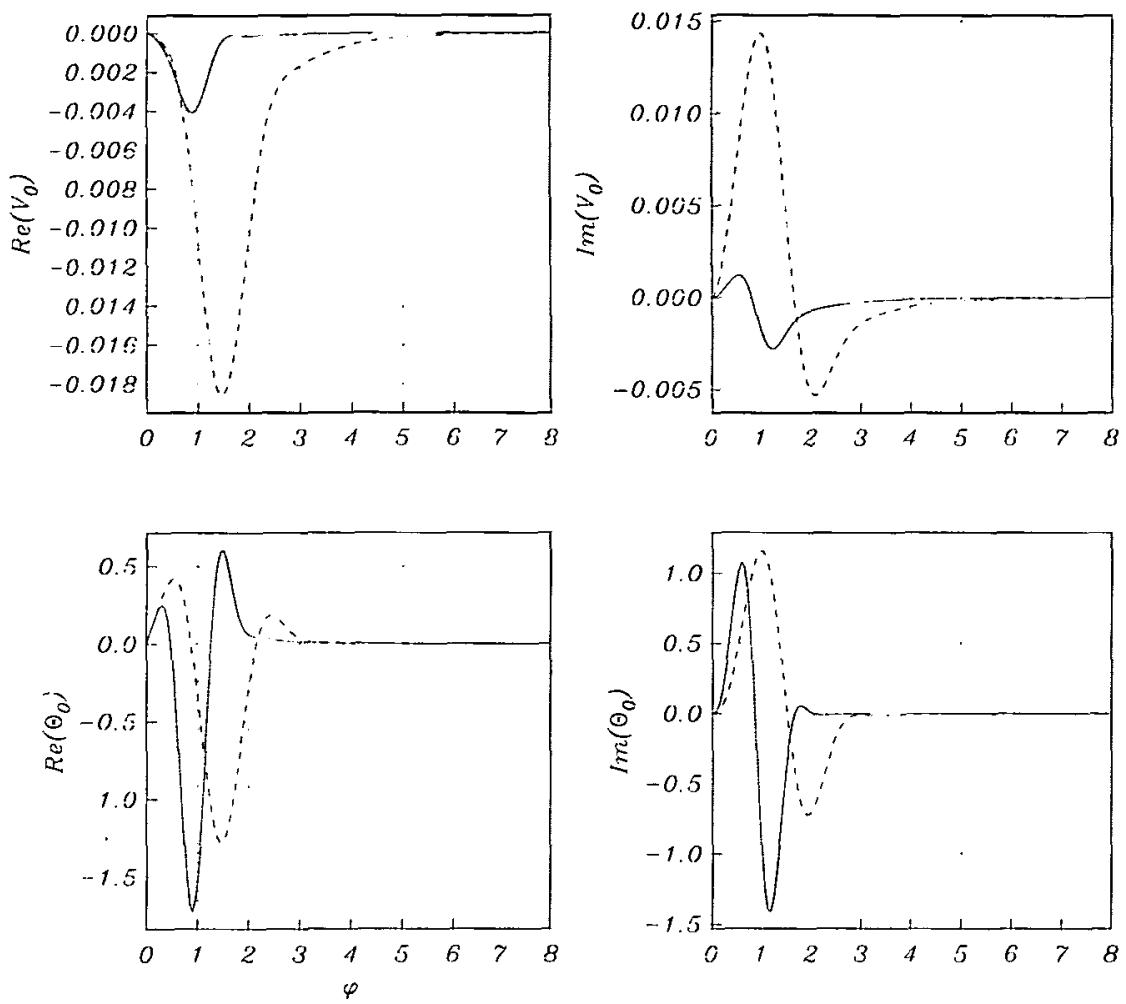

FIGURE 11. Eigenfunctions of the system (26)-(27) for wavenumbers $\alpha_{00}=0.2$ (dashed curve) and $\alpha_{00}=0.1$ (solidus).

from the Couette component of the flow, is increased. We have demonstrated that as this element grows the flow is stabilised to two-dimensional wave modes and these neutrally stable wave modes are driven to progressively shorter wavelengths. As such it is those modes relevant to the large Reynolds number-large wavenumber limits that are the last to be stabilised.

The modifications to the localized structure of the right-hand branch wave modes, first described by Mureithi et al. [16], as the level of shear is increased have been described. Thus as the wall shear is increased from its value for pure Poiseuille flow the neutral wave modes move from the centre of the channel to the upper boundary. A modified viscous wall bounded flow then emerges and the flow is finally rendered completely stable when the wall shear $u_{w}>2.9432$. This scenario of a re-stabilization of the flow by forcing the neutral wave-modes to within the vicinity of the rigid boundary is not unlike that which occurs in the problem of the stability of (unstratified) Poiseuille-Couette flow; see Cowley \& Smith [3] for details. 
This modified structure, described in Section 4.2 and Section 4.3, has implications for the nonlinear development of the right-hand-branch neutral wave modes. Indeed, in the regime $0<u_{w}<2$ the results of Denier \& Mureithi [6] are directly applicable to the current problem of stratified Poiseuille-Couette flow. Denier \& Mureithi [6] demonstrated that the nonlinear development of a single monochromatic wave in a strongly stratified boundary layer flow is governed by a wave/mean flow interaction in which the wave amplitude and the wave induced mean flow are of comparable sizes. In addition this nonlinear travelling wave bifurcates subcritically from the linear righthand-branch modes and is, as in the the case of linear wave mode considered in Mureithi et al. [16] and in the present paper, localized about the position of maximum streamwise velocity. Thus with only minor modifications the results of Denier \& Mureithi [6] can be applied to the current problem provided $0<u_{w}<2$ (so that the dominant wave motion is confined to a viscous layer located at the position of maximum streamwise velocity). However, as the level of wall shear is increased past the critical level of $u_{w}=2+O\left(\operatorname{Re}^{-3 / 8}\right)$ and the linear wave mode becomes localized at the upper boundary the results of [6] must be modified to account for the presence of the boundary. The modifications to the analysis of Denier \& Mureithi [6] which are required to fully understand the nonlinear evolution of these wall bounded wave modes is the subject of ongoing work, see [5].

\section{Acknowledgment}

All computations were carried out on a SGI Power Challenge operated by the South Australian Centre for Parallel Computing. We would like to thank an anonymous referee whose comments helped to improve the presentation of this paper.

\section{References}

[1] M. Abramowitz and I. A. Stegun (eds.), Handbook of Mathematical Functions (Dover, New York, 1965).

[2] N. D. Blackaby and M. Choudhari, "Inviscid vortex motions in weakly three-dimensional boundary layers and their relation with instabilities in stratified shear flows", Proc. R. Soc. Lond. A 440 (1993) 701-710.

[3] S. J. Cowley and F. T. Smith, "On the stability of Poiseuille-Couette flow: a bifurcation from infinity", J. Fluid Mech. 156 (1985) 83-100.

[4] J. W. Deardorff, "Gravitational instability between horizontal plates with shear", Phys. Fluids 8 (1965) 1027-1030.

[5] J. P. Denier, "Nonlinear wave interactions in stratified Poiseuille-Couette flow" (1998), in preparation.

[6] J.P. Denier and E. W. Mureithi, "Weakly nonlinear wave motions in a thermally stratified boundary layer", J. Fluid Mech. 315 (1996) 293-316. 
[7] P. G. Drazin and W. H. Reid, Hydrodynamic stability (C.U.P., 1979).

[8] K. Fujimura and R. E. Kelly, "Stability of unstably stratified shear flow between parallel plates", Fluid Dyn. Res. 2 (1988) 281-292.

[9] K. S. Gage, "The effect of stable thermal stratification on the stability of viscous parallel flows", $J$. Fluid Mech. 47 (1974) 1-20.

[10] K. S. Gage and W. H. Reid, "The stability of thermally stratified plane Poiseuille flow", J. Fluid Mech. 33 (1968) 21-32.

[11] A. P. Gallagher and A. McD. Mercer, "On the behaviour of small disturbances in plane Couette flow with a temperature gradient", Proc. Roy. Soc. Lond. A 286 (1965) 117-128.

[12] P. Hall, "Taylor-Görtler vortices in fully developed or boundary layer flows: linear theory", $J$. Fluid Mech. 124 (1982) 475-494.

[13] T. H. Hughes and W. H. Reid, "The stability of spiral flow between rotating cylinders", Phil. Trans. R. Soc. Lond. 263 (1968) 57-91.

[14] D. Koppel, "On the stability of flow of a thermally stratified fluid under the action of gravity", $J$. Math. Phys. 5 (1964) 963-982.

[15] J. W. Miles, "On the stability of heterogeneous shear flows", J. Fluid Mech. 10 (1961) 496-508.

[16] E. W. Mureithi, J. P. Denier and J. A. K. Stott, "The effect of buoyancy on upper branch TollmienSchlichting waves", IMA. J. Appl. Math. 58 (1997) 19-50.

[17] S. R. Otto and A. P. Bassom, "Weakly nonlinear stability of viscous vortices in three-dimensional boundary layers", J. Fluid Mech. 249 (1993) 597-618.

[18] P. Schäfer and H. Herwig, "Stability of plane Poiseuille flow with temperature dependent viscosity", Int. J. Heat Mass Transfer 36 (1993) 2441-2448.

[19] M. Tveitereid, "On the stability of thermally stratified plane Poiseuille flow", ZAMM 54 (1974) 533-540.

[20] O. V. Vasilyev and S. Paolucci, "Stability of unstably stratified shear flow in a channel under non-Boussinesq conditions", Acta Mech. 112 (1995) 37-58. 